\title{
PLASMA BRAIN NATRIURETIC PEPTIDE CONCENTRATIONS IN CHILDREN WITH EPILEPSY: THE EFFECT OF ANTIEPILEPTIC DRUGS
}

A. Garoufi ${ }^{1}$, E. Kalimeraki ${ }^{1}$, C. Tsendidis ${ }^{2}$, E. Katsarou ${ }^{3}$, D. Kyriakou ${ }^{1}$, E. Koemtzidou ${ }^{1}$, A. Marmarinos ${ }^{4}$, D. Gourgiotis ${ }^{4}$

${ }^{I}$ 2nd Department of Pediatrics, P\&A Kyriakou Childrens Hospital, University of Athens, Medical School, Athinai, ${ }^{2}$ Department of Endocrinology, General Hospital of Nicaea, Piraeus, ${ }^{3}$ Department of Neurology, $P \& A$ Kyriakou Childrens Hospital, ${ }^{4}$ Research Laboratories, 2 d Department of Pediatrics, P\&A Kyriakou Childrens Hospital, University of Athens, Medical School, Athinai, Greece

Background and aims: Plasma Brain Natriuretic Peptide (BNP) is recognized as a reliable marker for cardiac dysfunction. Recent studies postulate that intracerebral epileptic activity may trigger BNP secretion by heart or by brain. This prospective study aimed to evaluate BNP concentrations, before and after valproate sodium (VPA) or ox-carbamazepine (OX-CMZ) monotherapy, in children with idiopathic epilepsy.

Methods: BNP levels were determined in 59 (aged 4 to 15years, 29 boys) epileptic, otherwise healthy, children and 143 controls (matched for sex and age). BNP fragment was investigated in a fasting blood sample by enzyme immunoassay. In epileptic group the investigations performed before and at 8 and 20 months of VPA $(n=27)$ or OX-CMZ $(n=32)$ monotherapy. Children were at least 48 hours seizure-free. $\mathrm{P}<$ 0.05 was considered significant.

Results: Epileptic group had significantly lower BNP levels than controls before $(p=0.0003)$ and at 8 months $(\mathrm{p}=0.0001)$, but similar levels after 20 months therapy $(\mathrm{p}=0.12)$. Anticonvulsant use resulted in an increase of BNP only after 20 months therapy $(\mathrm{p}=0.0002)$. There were no significant differences at baseline, 8 and 20 months levels between VPA and OX-CMZ group $(\mathrm{p}=0.46, \mathrm{p}=0.21, \mathrm{p}=0.32)$. BNP levels had no significant correlation with gender $(\mathrm{p}=0.44)$, age group $(<6,6-12,>12$ years $)(\mathrm{p}=0.43)$, BMI $(\mathrm{p}=0.56)$ and BMI alterations during therapy $(\mathrm{p}=0.96)$ although $67.8 \%$ of children increased their body weight at 20 months.

Conclusions: Epileptic children have significantly lower BNP concentrations, after a seizure free interval, compared to non-epileptic. The long-term VPA or OX-CMZ monotherapy increase BNP at levels similar to those of healthy children. 